\title{
An Improved Clustering Using by Likely Attributable Function and Informed Selection in WSN for Science of Management and Engineering
}

\author{
Tarlan Motamedi nia ${ }^{1}$, Rohollah Omidvar ${ }^{2, *}$ and Elham Azarm ${ }^{3}$ \\ ${ }^{1}$ Institute for Management and Planning studies of Tehran, Iran \\ ${ }^{2}$ Shiraz Azad University, Iran Sama Computer School, Shiraz, Iran \\ ${ }^{3}$ Department of Computer Engineering, Shiraz Branch, Islamic Azad University of shiraz branch, shiraz, Iran
}

\begin{abstract}
Wireless sensors networks (WSNs) are traditionally composed of large number of tiny homogenous sensors nodes connected through a wireless network that gather data to be treated locally or relayed to the sink node through multi-hop wireless transmission. The low-energy adaptive clustering hierarchy (LEACH) protocol is one of the Famous protocols used in the wireless sensor networks (WSNs). The LEACH protocol in wireless sensor network suffers from many Bugs and many researchers proposed different methods to mitigate them. In this paper, we propose two ideas in a format for improving leach protocol. For Cluster head selection we used a Likely Attributable Function that in this function used from a factor. This factor that we called the informed selection factor helps to farther nodes not selection for cluster head. This significantly decreases the energy consumption and increases the lifetime of associated nodes. Simulation is conducted in using MATLAB results are analyzed for energy consumption.
\end{abstract}

Keywords: Wireless sensor network, Cluster head, Distance, Energy, Node, LEACH.

\section{INTRODUCTION}

Low Energy Adaptive Clustering Hierarchy (LEACH) protocol is widely used for the wireless networks which contain small battery powered devices, for example wireless sensor networks (WSN). When the battery power is drained in these devices/nodes then the network cannot be used and all the nodes spend most of the energy while transmitting the data. Therefore, to increase the lifespan of the network, each node has to do only minimal work for transmitting the data. LEACH protocol is widely used in WSN, because this protocol dissipates the energy in low level [1]. In hierarchical architecture sensing region is divided in to a number of clusters and a set of nodes are periodically elected as cluster head $(\mathrm{CH})$. Cluster head collects the data from non- $\mathrm{CH}$ nodes, aggregate it and then send it to the sink for further processing [2-4]. Clustering thus evenly distribute the energy load, reduce the energy consumption and increase the network life [4-9], [10-12]. In this paper presented an idea that the cluster head is selected according to the likely attributable intelligent algorithms. This paper is divided into sections as follows. Section 2 explains the related works. Section 3 describes the wireless sensor network model, Section 4 describes radio energy model, Section 5 explains LEACH protocol propose, simulation results are explored in Section 6 and finally the paper is concluded in Section 7.

Address correspondence to this article at the Shiraz Azad University, Iran Sama Computer School, Shiraz, Iran;

Tel: +989366757898;

E-mail: r.omidvar.uni@gmail.com

\section{RELATED WORK}

In hierarchical routing protocols whole network is divided into several clusters. One node in each cluster play leading rule. Cluster-head is the only node that can transmit to Base station in clustering routing protocols. This significantly reduces the routing overhead of normal nodes because normal nodes have to transmit to cluster-head only. Explanation of some hierarchical routing protocols is discussed in next subsections.

In a research [13] Heinzelman and al. have proposed a distributed clustering algorithm called Low-Energy Adaptive Clustering Hierarchy (LEACH), for routing in homogeneous wireless sensor networks. LEACH selects randomly the nodes cluster-heads and assigns this role to different nodes according to roundrobin management policy to ensure fair energy dissipation between nodes In order to reduce the value of information transmitted to the base station, the cluster-heads aggregate the data captured by the member nodes belonging to their own cluster, and then sends an aggregated packet to the base server. The LEACH protocol consists of two phases: The first is the set-up phase, and the second is the steady-state in the first phase, cluster heads are selected and clusters are formed, and in the next phase, the data transfer to the base server is held. During the first phase, the process of electing cluster heads is triggered to select future cluster heads. Thus, a predetermined fraction of nodes connected as cluster heads according either 0 or 1 . If the random number is less than a threshold function Ts then the node becomes a cluster head in the current round, otherwise the node $\mathrm{n}$ is expected to join the 
nearest cluster head in its neighborhood. The threshold is set as:

$T(n)=\left\{\begin{array}{c}\frac{T}{1-p\left(r \bmod \frac{1}{p}\right)} \text { if } n \in G \\ 0 \text { Otherwise }\end{array}\right.$

LEACH offers no guarantee about the placement or number of cluster heads. In [5], an enhancement over the LEACH protocol was proposed. The protocol, called LEACH-C, uses a centralized clustering algorithm and the same steady-state phase as LEACH. During the first phase of $\mathrm{LEACH}-\mathrm{C}$, each node sends information about its current location and its energy level to the base station. In addition to determining good clusters, the base station needs to ensure that the energy load is evenly distributed among all the nodes. To do this, the base station calculates the average node energy, and determines which nodes have energy below this average.

The proposed an improvement to the cluster-based $\mathrm{LEACH}$ routing protocol for greater energy efficiency in a WSN. In the proposed routing protocol, we consider the residual energy and geometric distance between candidate nodes and the $\mathrm{BS}$ to select $\mathrm{CH}$ nodes. It is clear that the $\mathrm{CH}$ node closer to the BS will consume less energy than other nodes because communication of data consumes the most energy in WSNs. In addition, we include the residual energy of the $\mathrm{CH}$ nodes and exchange cluster-head roles after a duration to balance energy consumption among the nodes in the WSN. Although the results of the simulation confirmed that LEACH-DE outperforms LEACH and $\mathrm{LEACH}-\mathrm{C}$ in lengthening WSN life time [16].

In this propose protocol analyses the effectiveness of LEACH protocol in cluster-head selection, and proposes an improved clustering algorithm. This new algorithm takes node's residual energy and location information into account, optimizes the selection method of the threshold for electing cluster-head, improves optimal cluster-head selection strategy that is normal nodes select the optimal cluster-head based on the cost function. On the basis of traditional LEACH protocol, this method proposes an energy balance algorithm optimizing cluster-head selection. This algorithm comprehensively considers the residual energy and distance factors, improves cluster-head election and the strategy of non-cluster head node selecting the optimal cluster-head. As is proved in the simulation result, the improved algorithm can effectively balance the network energy consumption, heighten system data transmission, and prolong the nodes and network life. The next focus is a combination of the improved algorithm and multi-hop routing, and paying more attention to the complexity of the algorithm and network overhead. Simulation results show that the improved protocol is better than LEACH in balancing node energy consumption, improving the efficiency of data transmission and prolonging the network life [15].

In one of research [17] introduce PEGASIS-LEACH $(P-L E A C H)$, a near optimal cluster-based chain protocol that is an improvement over PEGASIS and LEACH both. This protocol uses an energy-efficient routing algorithm to transfer the data in WSN.

In [18] presented on Multi-Hop LEACH energy efficient routing protocol for wireless Sensor networks, a method of routing is suggested based on both particle swarm optimization technique and V-LEACH protocol and multi-hop network design. To minimize energy dissipation in the transmission from cluster head to the base station, multi-hop communication has been used.

For some problems of the LEACH algorithm, the LEACH algorithm is improved in [19] from three aspects of the optimal number of the cluster head and uniform distribution and many jump communication, the improved algorithm through the simulation tests show that network node energy has been obviously saved.

Authors created an O-LEACH [20] Orphan nodes protocol, its role is to reduce the sensor nodes which do not belong the cluster. O-LEACH present two scenarios, the first scenario consists, a cluster member will be able to play the role of a gateway which allows the joining of orphan nodes. The second scenario consists, if in an area not covered, the number of orphan nodes is very important, if number of cluster member is superior to number of orphan nodes, a sub-cluster will be created.

In a paper [21], authors propose an improved multipath leach protocol, which uses at most one intermediate cluster head. The aim of our protocol is to extend the lifetime of the network and send more data compared to the original protocol. They used Castalia simulator to compare LEACH protocol and ICH-LEACH protocol by creating an application in the application layer. This application generates a packet every period of time, and then sends it to the lower layer.

\section{WIRELESS SENSOR NETWORK MODEL}

The operation of LEACH is including two sections. Each round begins with a set-up phase when the clusters are organized, followed by a steady-state phase when data are transferred from the nodes to the Base Station. During the set-up phase, each node normal chooses a random number between 0 and 1 . If the number is less than a threshold $T(n)$, the node 
becomes a cluster head for the current round. The threshold is set as equation 1. The following assumptions are made in the presented work.

- The Base Station is fixed and located far from the sensors.

- $\quad$ All nodes in the network are homogeneous and energy constrained.

- $\quad$ All nodes can transmit with enough power to reach the Base Station.

- $\quad$ All nodes can adjust their transmit power

- $\quad$ The nodes always have data to send to the Base Station and nodes located close to each other have correlated data.

\section{RADIO MODEL ENERGY}

We use the same radio model as stated in [5-13]. Matching to the radio energy dissipation model of Figure 1, the energy consumption of transmitting I-bit data over a distance $d$ is

$E_{T X}(l, d)=\left\{\begin{array}{l}l E_{\text {elec }}+l \varepsilon_{f S} d^{2} \text { if } d<d_{0} \\ l E_{\text {elec }}+l \varepsilon_{m p} d^{4} \text { if } d \geq d_{0}\end{array}\right.$

Where $d_{0}=\sqrt{\varepsilon_{f s} \varepsilon_{m p}}$ denotes the threshold distance, $E_{\text {elec }}$ represents the energy consumption in the electronics for sending or receiving one bit, and $\varepsilon_{f s} d^{2}$ and $\varepsilon_{m p} d^{4}$ is the amplifier energy that depends on the transmitter amplifier model.

The energy consumption of receiving l-bit data is

$E_{R X}(l)=l E_{\text {elec }}$

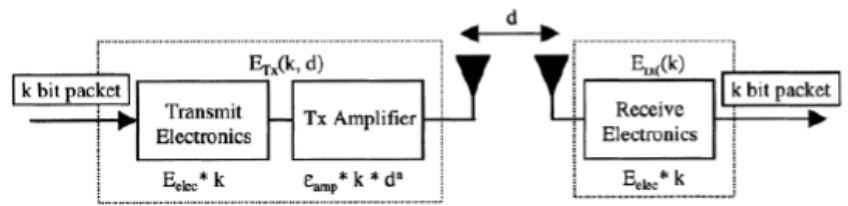

Figure 1: Radio energy dissipation model.

Here we use the typical values $E_{\text {elec }}=50$ $\mathrm{nJ} / \mathrm{bit}, \varepsilon_{f s}=10 \mathrm{pJ} / \mathrm{bit} / \mathrm{m}^{2}$ and $\varepsilon_{m p}=0.0013 \mathrm{pJ} / \mathrm{bit} / \mathrm{m}^{4}$. As noted previously, the cluster heads are responsible for aggregating their cluster members' data. The energy for data aggregation is set as $E_{D A}=5 \mathrm{~nJ} / \mathrm{bit} /$ signal.

\section{PROPOSED MODEL}

The proposed schemes aim to enhance the election method of cluster heads by minimizing power consumption of network nodes and informed choice of $\mathrm{CHs}$. The schemes take into consideration the drawbacks of the original LEACH, and accordingly try to minimize the energy consumption. The main idea of our proposed schemes is split into two stages. The first stage is Use Likely Attributable Function for cluster head election process. In the second stage, the informed selection factor has been also appended to the threshold equation. These stages are discussed as follows:

\section{(A) Stage One}

The first stage changes the basic election of cluster heads by use Likely Attributable Function in which it informed selection factor. In the $A B C$ onlookers are assigned to food sources by a stochastic rule, assuming a certain probability $p_{j}$ related to a fitness value $\mathrm{f}$ it $\left(x_{j}\right)$ of the configuration $x_{j}$ of the food source $\mathrm{j}$ [14]:

$P j=\frac{f i t\left(x_{j}\right)}{\sum_{k=1}^{S N} f i t\left(x_{j}\right)} \forall_{j} \in\{1, \ldots, S N\}$

Where $f i t\left(x_{j}\right)$ is the merits of each sensor node and $\sum_{k=1}^{S N} f i t\left(x_{j}\right)$ Competence of total nodes is Equation 4 called the Likely attributable function that we have used from this function to select the cluster head. This function makes use of the fitness of each node to all nodes in each round will be judged and the most suitable node is be selected as the cluster head. The threshold for the normal nodes to become cluster head can be evaluated by the following equation:

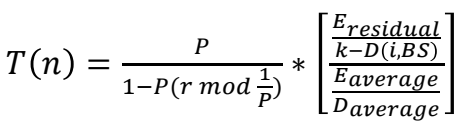

Where $E_{\text {residual }}$ is the residual energy of candidate node $\mathrm{i}$ at the current time, $D(i, B S)$ is the geographic distance from the candidate node to the $B S, k$ is the informed selection factor, $E_{\text {average }}$ is the average energy of network and $D_{\text {average }}$ is the average distance from total nodes to BS. K is factor Described in the next section. The average energy is calculated with equation 6 :

$E_{\text {average }}=\frac{E_{\text {total }}}{n}$

The average distance is calculated with equation 7 :

$D_{\text {average }}=\frac{\sum_{i=1}^{n} D(i, B S)}{n}$

The geometric distance between two nodes, $d(a, b)$ is computed as follows[17]

$|d(a, b)|=\sqrt{\left(x_{a}-x_{b}\right)^{2}+\left(y_{a}-y_{b}\right)^{2}}$

One of the drawbacks Leach protocol is cluster-head selection Based on probability that with this threshold this problem disappears. 


\section{(B) Stage Two}

In Leach protocol, selection of far nodes to BS, Caused consume a lot of energy, with applying a factor, we solving this problem. This factor called informed choice factor. This factor calculated in equation 9:

$$
k=D\left(\frac{n}{2}\right)
$$

Where $D(n)$ is the calculated in equation 1 .

$D(n)=\left[\operatorname{sort} \sum_{i=1}^{n} D(i, B S)\right.$, descend $]$

By applying this factor, as shown in Figure 2, the land is divided into two parts; the far section to BS with x2 shown in Figure 2, in lifetime network will not be selected as cluster head.

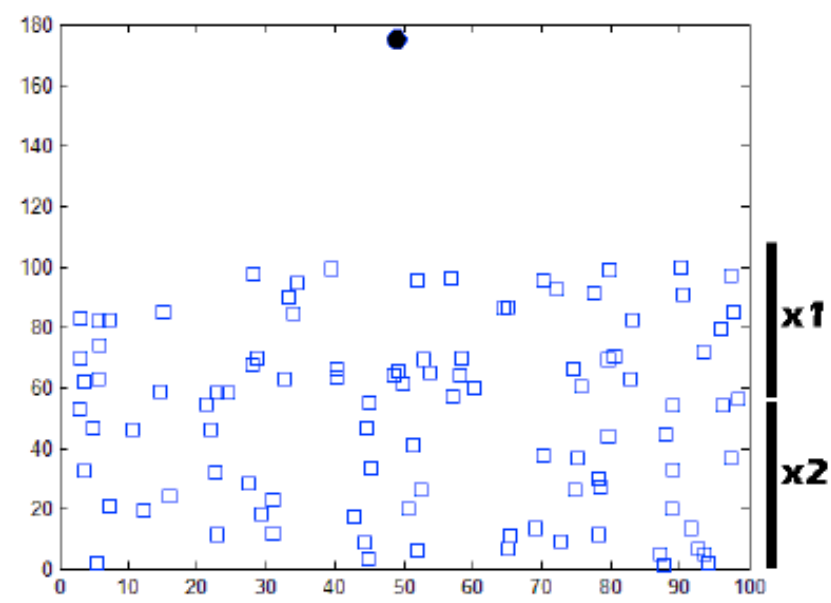

Figure 2: Model of cluster heads selection.

The selection of cluster heads in Leach protocol is shown in Figure 3.

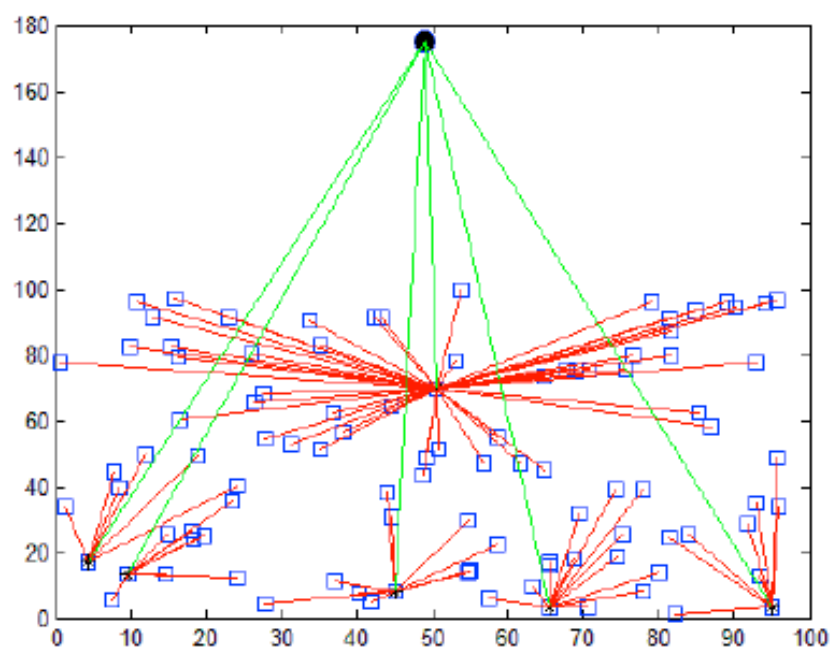

Figure 3: Model of cluster heads selection in LEACH.

According to Figure 3, cluster heads are selected from far distant nodes, hence the high consumption of energy, because the cluster heads with far distance for communication with the BS needs to consume more energy. The selection of cluster heads in Suggested method is shown in Figure 4, $\mathbf{5}$ and $\mathbf{6}$. This factor can be changed based on a multiple of even number, and causing the selection cluster heads from different parts of the earth, depending on your preference. To do this, in equation 9 , the denominator value will change depending on your selection.

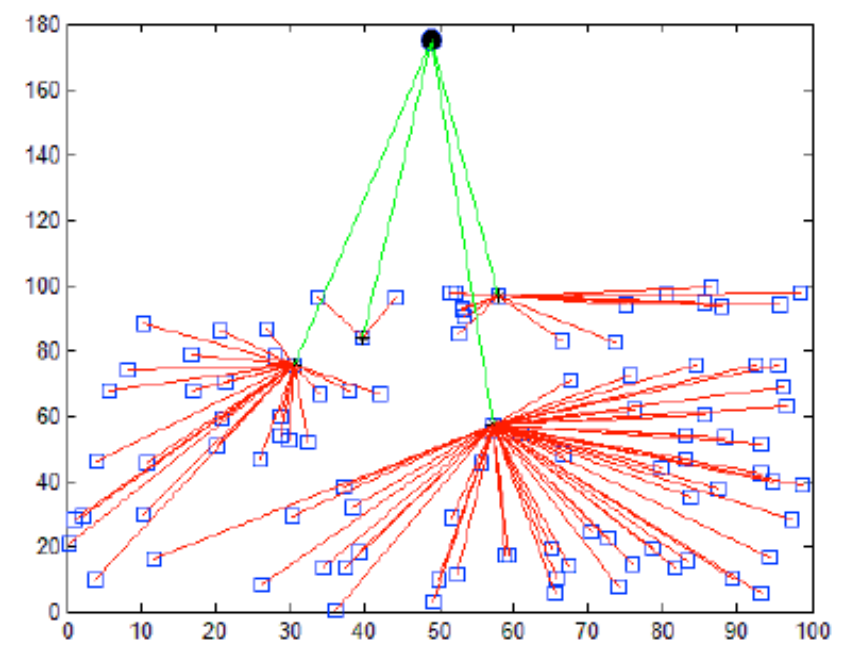

Figure 4: Model of cluster head selection in proposed method.

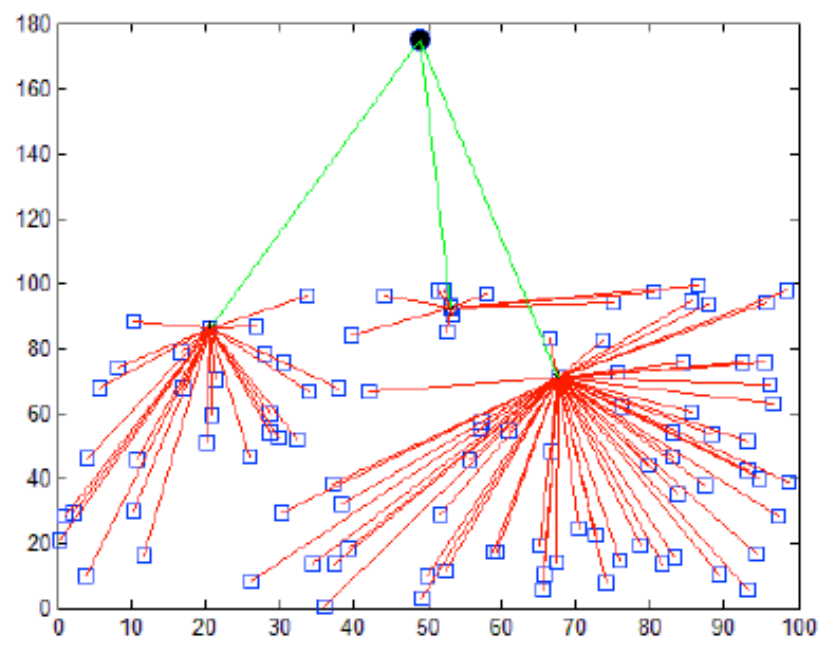

Figure 5: Model of cluster head selection in proposed method.

There are also new methods in this area, including the following articles:

In a paper [22], Authors have studied and designed an improved routing protocol for the field observation instruments network based on the LEACH protocol (FOI-LEACH). Firstly, the FOI-LEACH was proposed to mainly improve the LEACH protocol in three aspects: (1) the network nodes are heterogeneous and combined with the characteristics of field observation instrument networking. The residual energy and the rechargeable energy of nodes are added in the process of cluster head $(\mathrm{CH})$ election to reduce the risk of premature death of $\mathrm{CHs}$ and shortened network life 


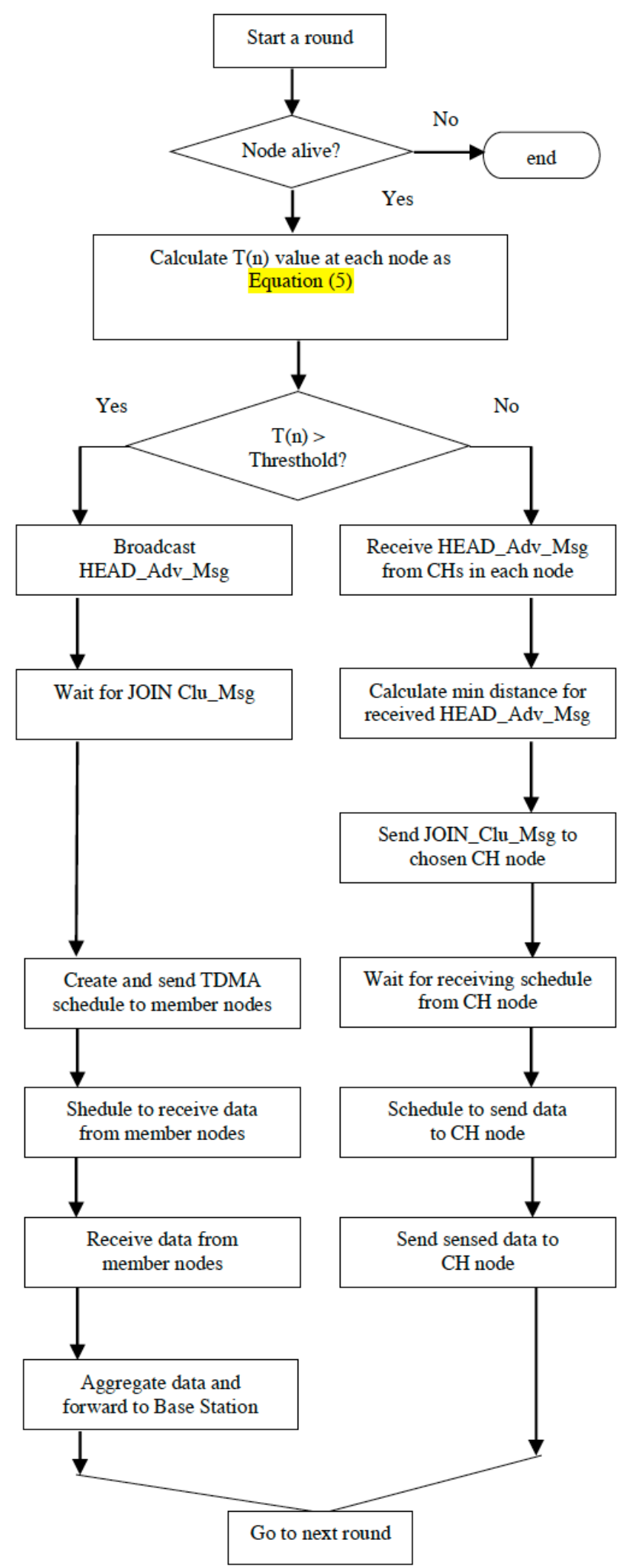

Figure 6: Flowchart for operation of propose method.

cycle caused by the selection of nodes with less energy as $\mathrm{CH}$. (2) In the process of cluster forming, the distance from $\mathrm{CH}$ to the base station (BS) and the residual energy of $\mathrm{CH}$ is considered when setting the cluster radius, to reasonably plan the cluster size and alleviate the "hot spot" problem. The nonuniform distribution of clusters in the network is enhanced to balance the total network energy consumption. (3) The autonomous zone-based multihop routing mechanism is adopted to solve the low reliability of data transmission caused by the poor quality of inter cluster communication links and premature death of nodes in long-distance transmission.

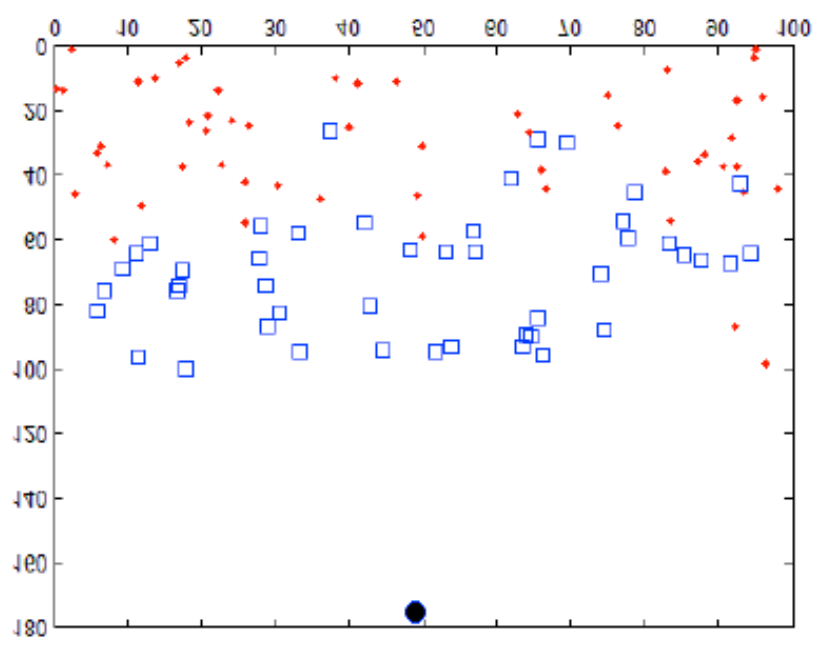

Figure 7: Number of nodes dead in the LEACH.

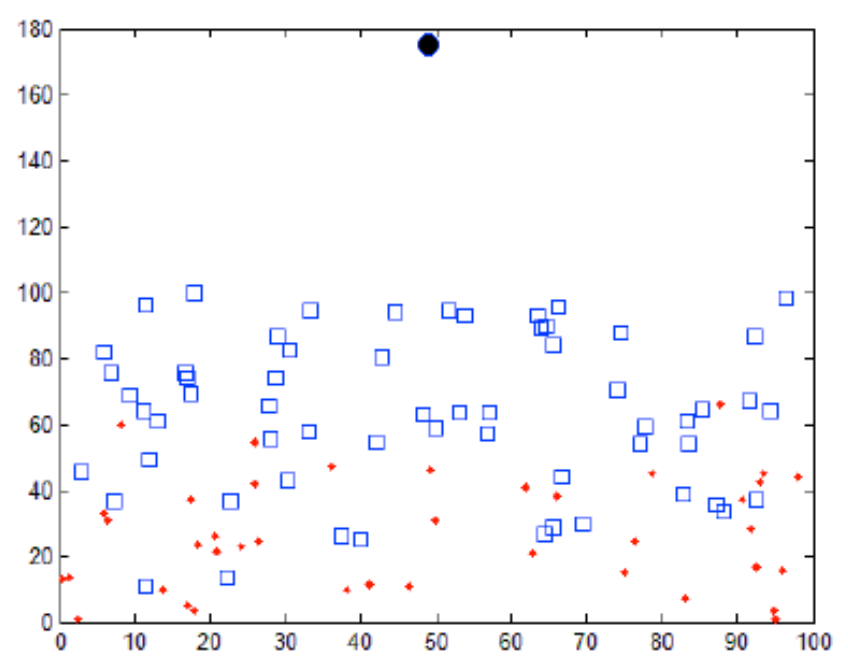

Figure 8: Number of nodes dead in the LEACH-C.

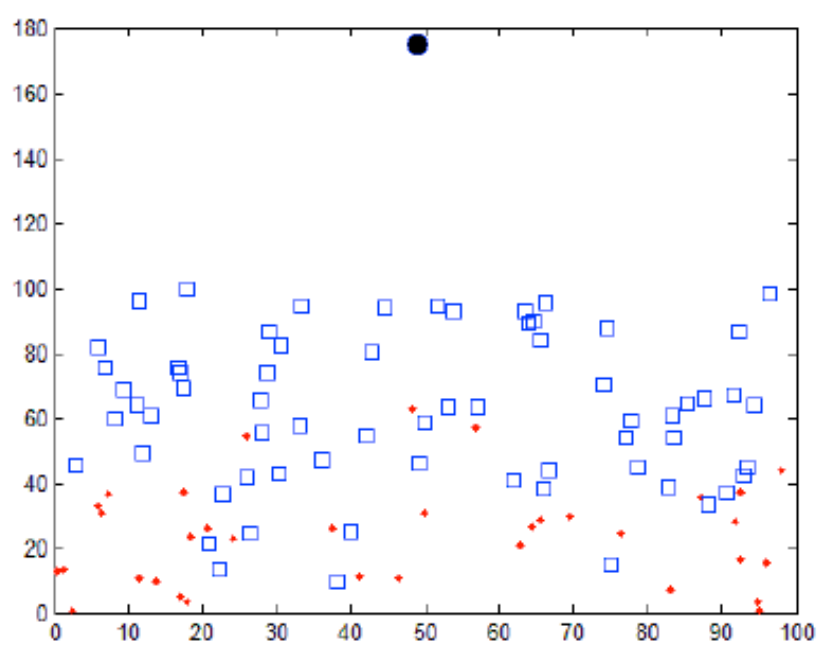

Figure 9: Number of nodes dead in the DE-LEACH. 


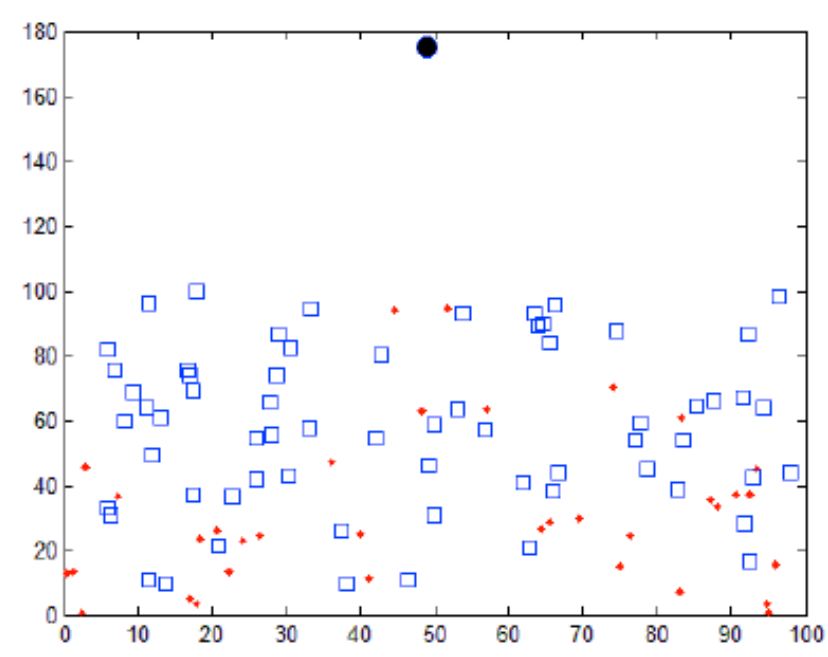

Figure 10: Number of nodes dead in the L-LEACH.

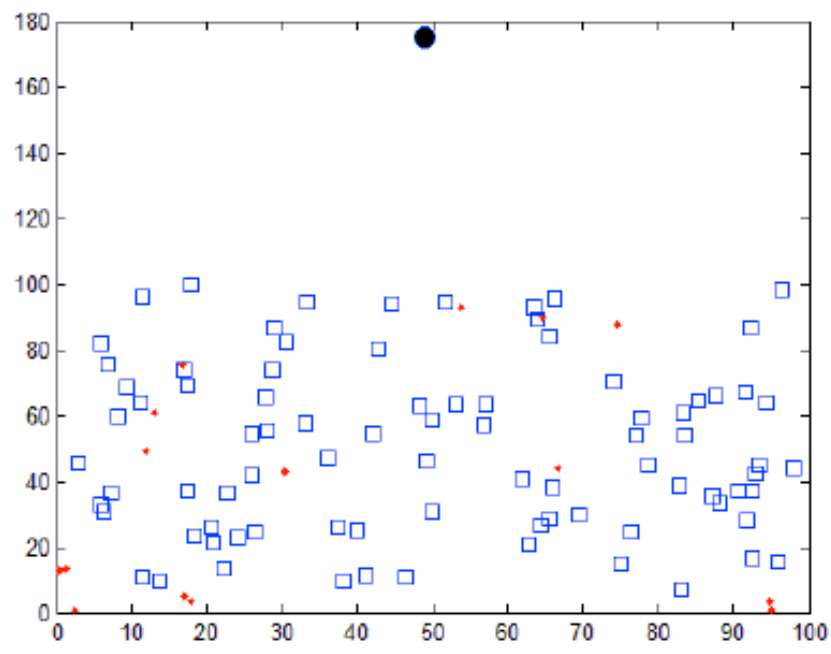

Figure 11: Number of nodes dead in the PROPOSE.

This paper [23] focuses on exploring most widely used Local Energy Aware Cluster Head (LEACH) protocol in the field of Underwater Wireless Sensor Network (UWSN). From the literature it can be found that most of the researchers have proposed energy efficient protocol for a single core machines. These protocols may work a efficiently in single core machines but exhibits low performance when applied to multi core machines hence this work proposes an Energy Efficient Parallel Local Energy Aware Cluster Head (EEPLEACH) protocol that evaluates and improves network performance considering energy metric in many core CPUs and GPUs. The proposed protocol is tested on Dual-core, Quad-core, Hexa-core and Octa-core machines.

This article [24] presents the design, analyses and implementation of the novel routing protocol for energy optimization based on LEACH for WSN. Network Lifetime is the major problem in various routing protocols used in WSN. In order to overcome that problem, proposed routing protocol is developed, which is a combination of Micro Genetic algorithm with
LEACH protocol. The proposed $\mu \mathrm{GA}-\mathrm{LEACH}$ protocol, strengthen the cluster head $(\mathrm{CH})$ selection and also reduce the energy consumption of the network when compared to existing protocols. This paper shows the improvement of network lifetime and energy consumption with the optimal $\mathrm{CH}$ selection based on a micro genetic algorithm.

\section{SIMULATIONS AND RESULT}

In this section, we evaluate the performance of our proposed algorithm through the simulations. We compare our proposed algorithm with LEACH based on performance metrics: total energy consumption, number nodes alive and system lifetime. Figures 7 to 11 show the vertical and horizontal axes of the length and width of the earth.

\section{(A). Parameter Setting}

We used MATLAB to test the modified LEACH protocol. Parameter settings are presented in Table 1.

Table 1: Parameter Setting

\begin{tabular}{|c|c|}
\hline Parameter & Value \\
\hline \hline Simulation area & $100 * 100$ \\
\hline Network size & 100 \\
\hline$E_{\text {elec }}($ Radio electronics energy $)$ & $50 \mathrm{NJ} / \mathrm{bit}$ \\
\hline $\mathrm{E}_{\text {amp }}($ Radio amplifier energy $)$ & $100 \mathrm{PJ} / \mathrm{bit} / \mathrm{m} 2$ \\
\hline $\mathrm{E}_{\mathrm{fs}}($ Radio free space $)$ & $0.013 \mathrm{pJ} / \mathrm{bit} / \mathrm{m} 4$ \\
\hline $\mathrm{E}_{\text {init }}$ (Initial energy of node $)$ & $1 \mathrm{~J}$ \\
\hline Energy model & Battery \\
\hline Packet size & 200 \\
\hline Base station at & 49,175 \\
\hline
\end{tabular}

Indicates the number of nodes and the $X$ axis represents the lifetime of the nodes in the WSN according to the simulation time.

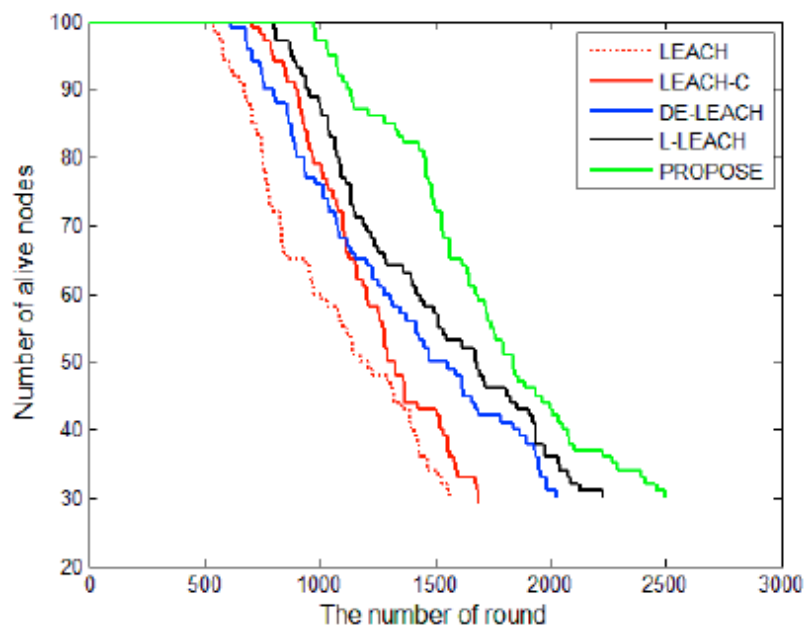

Figure 12: Number of nodes alive during the simulation time. 


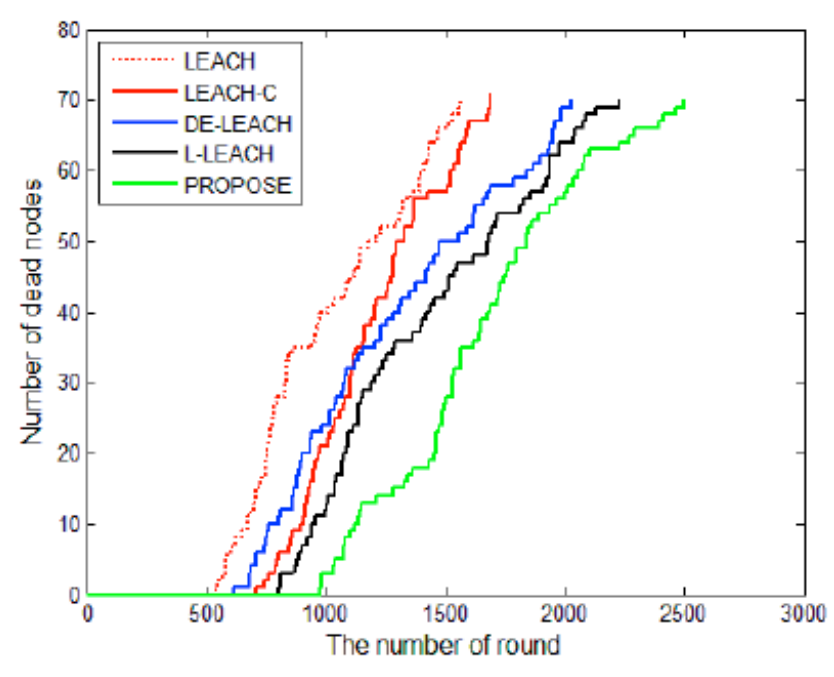

Figure 13: Number of nodes dead during the simulation time.

Figure 13 shows the percentage of dead nodes during the simulation time. It is obvious that propose algorithm achieved better energy efficiency and prolonged the WSN lifetime compared to LEACH and other methods. The results are shown in Figure 15; nodes in propose algorithm have a longer energy lifetime than with LEACH and other methods. According to Figure 14, the death of the first node in the proposed method is much higher than LEACH and other methods. The results showed that the mechanism of the proposed method was effective in creating a sensor network with a longer lifespan. The reason for this claim is that the proposed method was able to store more internal battery energy of the sensors during the life of the network, and this was due to the way the proposed method used the sensors. It can be said that the proposed method could work in such a way that the sensors consume less energy for their work. The results show that at any given time in the proposed method diagram, it always had more live sensors and always had fewer burned sensors than other methods.

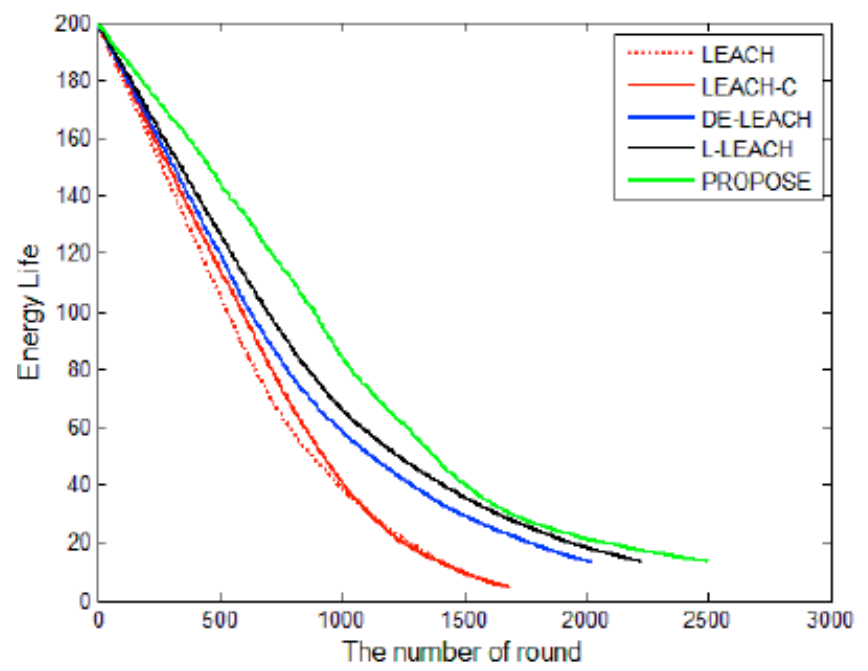

Figure 14: energy lifetime.

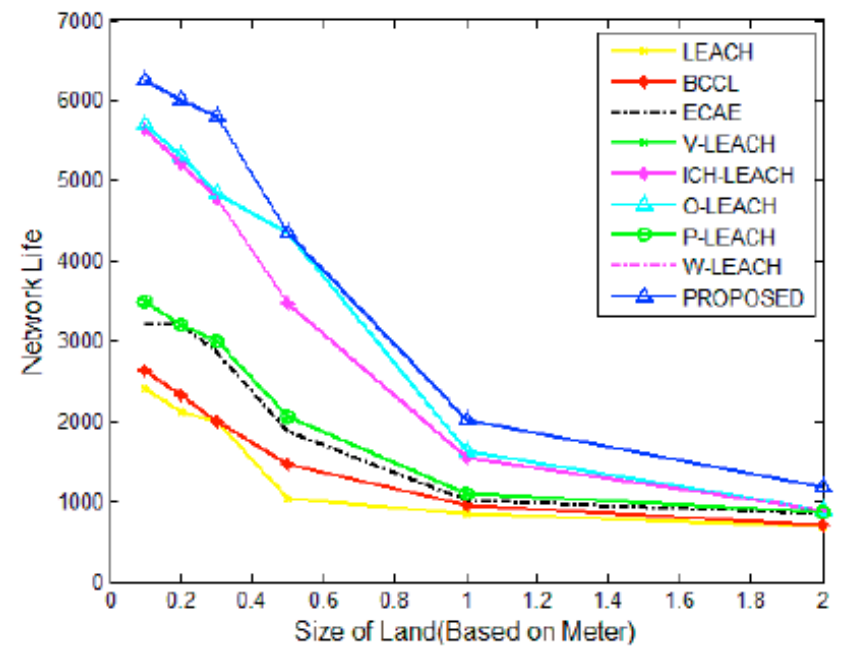

Figure 14: Number of nodes dead during the simulation time.

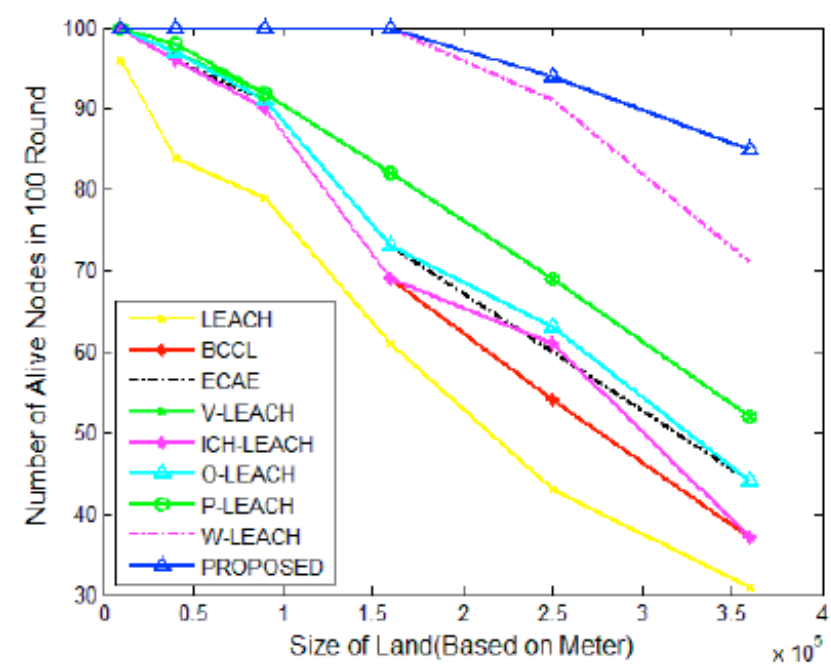

Figure 15: Number of nodes dead during the simulation time.

\section{CONCLUSION}

At the beginning of this article, we examined Leach's weaknesses and then presented this article based on that. The purpose of this paper is to provide a targeted mechanism to cover the weaknesses of the Leach protocol that can optimize the life of the sensor network. In this paper, we propose an improvement to the cluster-based LEACH routing protocol for greater energy efficiency in a Wireless Sensor Network. This paper introduced two idea of LEACH protocol. In the proposed routing protocol, the first idea we consider a Likely Attributable Function to select $\mathrm{CH}$ nodes. In second idea, we applied a factor in the cluster heads selection in the function proposed, than Cluster head be selected in the area where we wanted. Many simulation experiments were conducted to evaluate the significance of our proposed approach. Although the results of the simulation confirmed that method propose outperforms LEACH in lengthening Wireless Sensor Network lifetime, the number of member nodes in clusters, the duration of the steady-state phase, and 
How to the optimal selection of the cluster head in different area using change of informed selection factor will be researched in the future.

\section{REFERENCES}

[1] M. Madheswaran, RN. Shanmugasundaram, "Enhancements of Leach Algorithm For Wireless Networks: A Review", Ictact Journal on Communication Technology 2013; 4(4): 1

[2] K. Sohrabi and J. Pottie, "Protocols for self-organization of a wireless sensor network," IEEE Personal Commun. 2000; 7(5): $16-27$.

https://doi.org/10.1109/98.878532

[3] K. Akkaya and M. Younis, "A Survey on Routing Protocols for Wireless Sensor Networks," Ad Hoc Networks 2005; 3(3): 325-349.

https://doi.org/10.1016/j.adhoc.2003.09.010

[4] J. Al-Karaki, and A. Kamal, "Routing Techniques in Wireless Sensor Networks: A Survey," IEEE Communications Magazine, 2004; 11(6): 6-28. https://doi.org/10.1109/MWC.2004.1368893

[5] W. Heinzelman, A. Chandrakasan, and H. Balakrishnan, "An application specific protocol architecture for wireless microsensor networks," IEEE Transactions on Wireless Communications 2002; 1(4): 660-670 https://doi.org/10.1109/TWC.2002.804190

[6] Liang C, Kuo Y, Huang and J. Da Lin, "An energy efficient routing scheme in wireless sensor networks," In Advanced Information Networking and Applications-Workshops, 2008. AINAW 2008. 22nd International Conference on, pp. 916-921. IEEE, 2008. https://doi.org/10.1109/WAINA.2008.199

[7] J. Zheng and A. Jamalipour, "Wireless Sensor Networks: A Networking Perspective," a book published by A John \& Sons, Inc, and IEEE, 2009.

[8] S. Lindsey and C. Raghavendra, "PEGASIS: Power-Efficient Gathering in Sensor Information Systems," IEEE Aerospace Conf. Proc. 2002; 3: 9-16, pp. 1125-30.

[9] Younis, Ossama, and S. Fahmy. "HEED: a hybrid, energy-efficient, distributed clustering approach for ad hoc sensor networks." Mobile Computing, IEEE Transactions on 2004; 3(4): 366-379. https://doi.org/10.1109/TMC.2004.41

[10] A. Manjeshwar and D. P. Agrawal, " TEEN: A routing protocol for enhanced efficiency in wireless sensor networks," Proceedings IPDPS ' 01, San Francisco, CA, 2001; pp. 2009-2015

[11] Manjeshwar and DP. Agrawal, " APTEEN: A hybrid protocol for efficient routing and comprehensive information retrieval in wireless sensor networks, " Proceedings IPDPS' 01, San Francisco, CA, 2001; pp. 2009-2015. https://doi.org/10.1109/IPDPS.2002.1016600
[12] S. Bandyopadhyay and E. Coyle, "An energy efficient hierarchical clustering algorithm for wireless sensor networks," in Proceeding of INFOCOM 2003, vol. 3, San Francisco, CA, Apr. 2003; pp. 1713-1723

[13] W. Heinzelman, "Application-specific protocol architectures for wireless networks," Ph.D. dissertstion, Mass. Inst. Technol., Cambridge, 2000

[14] B. Akay, D. Karaboga, A modified artificial bee colony algorithm for real-parameter optimization. Information Sciences, 2010. https://doi.org/10.4249/scholarpedia.6915

[15] Q. Liao, H. Zhu," An Energy Balanced Clustering Algorithm Based on LEACH Protocol"," Proceedings of the 2nd International Conference On Systems Engineering and Modeling", Published by Atlantis Press, Paris, France 2013. https://doi.org/10.2991/icsem.2013.15

[16] N. Duy tan, L. Han, N. Dinh Viet, M. Jo, "An Improved LEACH Routing Protocol For Energy-Efficiency of Wireless Sensor Networks", "Smart Computing Review", 2012. https://doi.org/10.6029/smartcr.2012.05.006

[17] A. Razaque, M. Abdulgader, Ch. Joshi, P-LEACH: Energy efficient routing protocol for Wireless Sensor Networks, Systems, Applications and Technology Conference (LISAT), 2016 IEEE Long Island, 2016.

https://doi.org/10.1109/LISAT.2016.7494137

[18] A. Singh, Sh. Rathkanthiwar, S. Kakde, LEACH based-energy efficient routing protocol for wireless sensor networks, IEEE, 10.1109/ICEEOT.2016.7755602, 2016. https://doi.org/10.1109/ICEEOT.2016.7755602

[19] Sh. Xia, M. Tan, Zh. Zhao, T. Xiang, Improvement for LEACH Algorithm in Wireless Sensor Network, Springer, International Conference of Young Computer Scientists, 2016.

https://doi.org/10.1007/978-981-10-2053-7 26

[20] W. Jerbi, A. Guermazi, H. Trabelsi, O-LEACH of Routing Protocol for Wireless Sensor Networks, 13th International Conference on Computer Graphics, Imaging and Visualization (CGiV), 2016; pp. 399-404. https://doi.org/10.1109/CGiV.2016.84

[21] B. Mohamed Taj, M. Kbir, ICH-LEACH: An enhanced LEACH protocol for wireless sensor network, IEEE, Advanced Communication Systems and Information Security (ACOSIS), International Conference on, 2017.

[22] X. Deng, H. Al-Neshmi, Design and Improvement of Routing Protocol for Field Observation Instrument Networking Based on LEACH Protocol, Journal of Electrical and Computer Engineering, Volume 2020, Article ID 8059353, 2020. https://doi.org/10.1155/2020/8059353

[23] H. Davanagere, Energy Efficient Parallel LEACH Protocol for Underwater Wireless Sensor Network, International Journal of Information Technology 2020; 1(1): 1-8.

[24] M. Radhika, P. Sivakumar, Energy optimized micro genetic algorithm based LEACH protocol for WSN, Springer Wireless Networks 2021; 27: 27-40. https://doi.org/10.1007/s11276-020-02435-8

Received on 26-09-2021 Accepted on 01-12-2021 Published on 31-12-2021

DOI: https://doi.org/10.31875/2409-9848.2021.08.10

(C) 2021 Motamedi nia et al.; Zeal Press

This is an open access article licensed under the terms of the Creative Commons Attribution Non-Commercial License (http://creativecommons.org/licenses/by-nc/3.0/) which permits unrestricted, non-commercial use, distribution and reproduction in any medium, provided the work is properly cited. 